\title{
CONOCIMIENTOS Y HÁBITOS DE EXPOSICIÓN SOLAR EN COMERCIANTES AMBULANTES DE UN MERCADO DE CHICLAYO, PERÚ
}

\author{
KNOWLEDGE AND HABITS OF SOLAR EXPOSURE IN STREET TRADERS OF A \\ MARKET IN CHICLAYO, PERU
}

\author{
Sherly Raquel Sempértegui-Ruiz', Carol Jennifer del Milagro Nuñez-Campos', Maxi Andrea Bustios-Ahumada', \\ Arenas-Piscoya Andrés Eduardo', Cynthia Lizeth Estela-Moreto², Winston Maldonado-Gómez ', \\ Sebastian Iglesias-Osores ${ }^{3}$, Alain Monsalve-Mera'
}

\begin{abstract}
RESUMEN
Objetivo: Determinar los conocimientos y hábitos de exposición solar en comerciantes ambulantes alrededor de un mercado de Chiclayo. Métodos: Se aplicó un cuestionario a 291 comerciantes ambulantes ubicados alrededor del mercado Modelo de Chiclayo, que evaluaron los hábitos de exposición solar, medidas de foto protección, antecedentes de quemaduras solares y cáncer de piel, asi como conocimientos de radiación ultravioleta y fuentes de información. Resultados: Las edades fueron, 18-25 (42,3\%), 36-54 (38,1\%), 55 -78 (19,6\%). En nivel de educación, el 13,1\% tiene primaria completa, el $32,6 \%$ tiene secundaria completa y el $8,2 \%$ superior completa. Al momento del estudio el $71,9 \%$ llevaban más de 3 años trabajando como comerciantes ambulantes. Los fototipos de los participantes fueron fototipo I $(0,3 \%)$, fototipo II $(4,5 \%)$, fototipo III $(24,7 \%)$, fototipo IV $(48,8 \%)$, fototipo V $(19,6 \%)$, fototipo VI $(2,15)$. Un $90 \pm 3,5 \%$ reconoce que causa cáncer de piel, un 53,6\% reconoce que una característica del envejecimiento de la piel por radiación son las manchas y verrugas, un $56 \%$ respondió que la radiación solar causa cataratas, un $52,92 \%$ respondió que la gente de piel blanca es más sensible a los rayos del sol. Un $48,5 \%$ de ellos declararon exponerse más de 6 horas a los rayos solares, un $24,7 \%$ se expone de 3 - 6 horas al día, mientras que el 8,6\% registraron el menor tiempo de exposición solar ( 0 - 2 horas). El $75,3 \%$ no utiliza bloqueador solar. Conclusión: Los conocimientos y hábitos de exposición solar en los comerciantes ambulantes son de regular a adecuado y los hábitos deficientes.
\end{abstract}

Palabras clave: Exposición a la radiación; Rayos ultravioleta; Protectores solares (fuente: DeCS BIREME).

\section{ABSTRACT}

Objective: To determine the knowledge and habits of solar exposure in street vendors around a Chiclayo market. Methods: A questionnaire were applied to 291 street vendors located around the Chiclayo Model market, who evaluated the habits of sun exposure, photo protection measures, a history of sunburn and skin cancer, as well as knowledge of ultraviolet radiation and sources of information. Results: The ages were, 18-25 (42.3\%), 36-54 (38.1\%), $55-78$ (19.6\%). At the educational level, 13.1\% have a complete primary, $32.6 \%$ have a complete secondary and $8.2 \%$ have a complete superior. At the time of the study, $71.9 \%$ had been working as street vendors for more than 3 years. The phototypes of the participants were phototype I (0.3\%), phototype II (4.5\%), phototype III (24.7\%), phototype IV (48.8\%), phototype V (19.6\%), phototype VI (2.15). $90 \pm 3.5 \%$ recognize that it causes skin cancer, $53.6 \%$ acknowledge that a characteristic of skin aging by radiation is spots and warts, $56 \%$ answered that solar radiation causes cataracts, $52.92 \%$ He replied that white-skinned people are more sensitive to the sun's rays. $48.5 \%$ of them declared that they were exposed to sunlight for more than 6 hours, $24.7 \%$ were exposed to 3-6 hours a day, while $8.6 \%$ had the shortest time of sun exposure ( 0 - 2 hours). $75.3 \%$ do not use sunscreen. Conclusion: The knowledge and habits of sun exposure in street traders are from fair to adequate and poor habits.

Key words: Radiation exposure; Ultraviolet rays; Sunscreening agents (source: MeSH NLM).

\footnotetext{
'Escuela de Medicina, Universidad Católica Santo Toribio de Mogrovejo, Chiclayo-Perú.

${ }^{2}$ Hospital Regional Lambayeque, Lambayeque-Perú.

${ }^{3}$ Facultad de Ciencias Biológicas, Universidad Nacional Pedro Ruiz Gallo, Lambayeque-Perú.
}

Citar como: Sherly Raquel Sempértegui-Ruiz, Carol Jennifer del Milagro Nuñez-Campos, Maxi Andrea Bustios-Ahumada, Andrés Eduardo Arenas-Piscoya, Cynthia Lizeth Estela-Moreto, Winston Maldonado-Gómez, Sebastian Iglesias-Osores, Alain Monsalve-Mera. Conocimientos y hábitos de exposición solar en comerciantes ambulantes de un mercado de Chiclayo, Perú. Rev. Fac. Med. Hum. Abril 2020; 20(2):261-267. DOI 10.25176/RFMH.v20i2.2899 


\section{INTRODUCCIÓN}

La radiación ultravioleta es el principal factor de riesgo en el desarrollo del cáncer de piel( ${ }^{(1)}$. Los estilos de vida conllevan a mayores exposiciones a la radiación ultravioleta, aumenta el riesgo de desarrollar cáncer de piel como lo es el caso de los vendedores ambulantes ${ }^{(2)}$. Los cánceres de piel se definen como células malignas o benignas ${ }^{(3)}$, el cáncer de piel es la neoplasia maligna más frecuente en el mundo y su prevalencia se ha elevado en los últimos años a nivel mundial ${ }^{(4)}$. Se estima que uno de cada cinco estadounidenses desarrollará cáncer de piel durante su vida y que una persona morirá de melanoma cada hora del día ${ }^{(3)}$. En Perú, el cáncer de piel ocupa el cuarto lugar en casos nuevos diagnosticado (2010$2012)^{(5)}$. Según datos de la Vigilancia Epidemiológica de Cáncer realizada en Perú en el 2016, el cáncer de piel ocupó el segundo lugar más frecuente en varones y el tercero en mujeres (MINSA). La luz solar es responsable de muchos efectos biológicos esenciales para la vida. Sin embargo, cuando es excesivo, puede presentar riesgos para la salud humana al causar quemaduras solares, foto envejecimiento y foto carcinogénesis ${ }^{(6)}$. La exposición al sol está relacionada con el desarrollo de todos los tipos de $\mathrm{SC}$, tanto melanoma como no melanoma ${ }^{(7)}$.

La radiación solar está compuesta por la luz visible, la infrarroja y la ultravioleta (UV). Los efectos crónicos indeseables de la radiación solar se deben primordialmente al espectro comprendido entre los 290 y $400 \mathrm{~nm}$ de longitud (UV). Estos incluyen quemaduras, carcinogénesis, inmunosupresión, cataratas oculares y foto envejecimiento. Estudios epidemiológicos han demostrado que la exposición solar y la sensibilidad UV de la población son los principales factores de riesgo para el desarrollo de neoplasias cutáneas ${ }^{(3,6-10)}$.

Las actividades de la vida diaria se llevan a cabo al aire libre, como el comercio informal en vendedores ambulantes, que es una realidad en todas las ciudades de Latinoamérica. La importancia de la práctica de medidas de protección solar en estas personas expuestas a períodos prolongados de radiación UV disminuye la morbilidad de padecer algún tipo de las lesiones y enfermedades mencionadas ${ }^{(11)}$.

El comportamiento de la morbimortalidad por cáncer de piel observado en estos estudios no conlleva a que es un importante problema de salud pública, a pesar de no conocerse su real prevalencia e incidencia, los nuevos casos de cáncer se han incrementado en forma sistemática. Lo anterior pone en manifiesto el carácter imprescindible de las campañas de prevención primaria, destinadas a reducir la exposición solar, el principal factor de riesgo modificable, y la promoción de la detección precoz del cáncer de piel|(6,11). Los objetivos del trabajo fueron evaluar el grado de conocimiento y los hábitos, que tienen los comerciantes ambulantes de un mercado de Lambayeque, Perú, acerca de la exposición solar y su relación con cáncer de piel, así como el conocimiento de las distintas modalidades de protección solar. También, se evaluaron conceptos y conductas erradas relacionadas con la protección solar.

\section{MÉTODOS}

\section{Diseño}

Estudio prospectivo de corte transversal.

\section{Población, tamaño de muestra}

291 comerciantes ambulantes ubicados alrededor del mercado Modelo de Chiclayo. Se trabajó con toda la población ubicada en el asiento geográfico de recolección de datos en el momento de la recolección de los datos.

\section{Procedimientos, variables e instrumentos}

El instrumento utilizado por los autores, fue aplicado a los comerciantes ambulantes que venden sus productos fuera del mercado Modelo de Chiclayo, Lambayeque durante la primera semana del mes de setiembre del año 2017. El horario de recolección de datos fue entre las 10:00 am y las 2:00 pm; lo cual garantizaba la necesidad de uso de material de protección solar. El encuestador constató en el terreno la información referente a los medios de protección solar referidos, procedió a repreguntar en caso de no coincidir. Se entrevistaron a todos los ambulantes presentes en el día planteado para la recolección de datos. Los ambulantes se encontraban ubicados alrededor del mercado, delimitado el espacio alrededor hasta dos cuadras del perímetro del mismo. Se evaluaron los hábitos de exposición solar, medidas de foto protección, antecedentes de quemaduras solares y cáncer de piel, así como conocimientos de radiación ultravioleta y fuentes de información. El instrumento utilizado fue adaptado de otro instrumento usado en un estudio realizado en el Centro Histórico de Trujillo ${ }^{(12)}$. A su vez, esta adaptación paso por una validación de juicio por expertos.

\section{Análisis estadístico}

Se usó estadística descriptiva, representando las variables cualitativas usando frecuencias absolutas y relativas; asimismo, para las variables cuantitativas se utilizaron las medidas de tendencia central y dispersión, previa evaluación de la normalidad usando la prueba de Kolmogorov-Smirnov. Todos los análisis estadísticos se ejecutaron haciendo uso del software estadístico InfoStat.

\section{Cuestiones éticas}

El proyecto fue aprobado por el Comité de Ética e Investigación de la Facultad de Medicina de la 
Universidad Católica Santo Toribio de Mogrovejo, además se aplicó un consentimiento informado el cual fue firmado por los participantes.

\section{RESULTADOS}

Se encuestó a un total de 291 comerciantes ambulantes del mercado Modelo de Chiclayo. Los rangos etarios distribuyeron de entre los rangos 18-25 (42,3\%), 36-54 (38,1\%), 55 -78 (19,6\%). Según el nivel de educación, el $13,1 \%$ tiene primaria completa, el $32,6 \%$ tiene secundaria completa y el $8,2 \%$ superior completa. Al momento del estudio el 71,9\% llevaban más de 3 años trabajando como comerciantes ambulantes.

Se estudiaron también los fototipos de piel de los ambulantes que trabajan alrededor de un mercado, en la que se observó que la siguiente frecuencia: fototipo I $(0,3 \%)$, fototipo II $(4,5 \%)$, fototipo III $(24,7 \%)$, fototipo IV $(48,8 \%)$, fototipo V $(19,6 \%)$, fototipo VI $(2,15 \%)$.

En la Tabla 3, se muestran los resultados de las preguntas dirigidas sobre las nociones de los comerciantes en relación con los efectos causados por la radiación solar. Las respuestas más relevantes en el ítem enfermedad por exposición solar prolongada son que un $90 \pm 3,5 \%$ reconoce que causa cáncer de piel, un 53,6\% reconoce que una característica del envejecimiento de la piel por radiación son las manchas y verrugas, en el ítem de daño a los ojos por exposición solar un 56\% respondió que la radiación solar causa cataratas, también un 52,92\% respondió que la gente de piel blanca es más sensible a los rayos del sol, así como cáncer de piel (90\%).

El 59,5 \% de los comerciantes (173/291) señaló acudir a laborar de lunes a domingo, un 39,4 \% trabaja de 5-6 días a la semana, y un 4,1\% trabaja 4 o menos días a la semana. Aproximadamente un (48,5\%) de ellos declararon exponerse más de 6 horas, un 24,7 $\%$ se expone a los rayos solares de $3-6$ horas al día, mientras que el 8,6\% registraron el menor tiempo de exposición solar (0 - 2 horas). La mayoría de los comerciantes no utiliza bloqueador solar $(75,3 \%)$, solo 72 comerciantes utilizaban protector solar al momento de ser encuestados. Solo el $24,7 \%$ de los encuestados (72) manifestó utilizar este producto como medida de fotoprotección; de ellos, el 63,9\% (68) manifestaron que un familiar les recomendó su uso, el 94,4\% lo usa de 1-2 veces al día, el 38,9\% solo se lo aplica cuando va a laborar, 52,8 \% (38) lo usa en cara, cuello, orejas y manos, y la mitad de ellos (50\%) lo obtiene de un catálogo (Tabla 4).

Los hábitos más comunes utilizados como práctica de fotoprotección fueron utilizar sombrillas (45\%) y pantalones (61\%), mientras que los menos frecuentes fueron el uso de lentes (60\%) y gorras (27\%) (Tabla 5).

Tabla 1. Características sociodemográficas de los comerciantes ambulantes de un mercado en Lambayeque, Chiclayo - setiembre, 2017.

\begin{tabular}{|c|c|c|c|}
\hline \multicolumn{2}{|c|}{ Características sociodemográficas } & $n^{\circ}$ & $\%$ \\
\hline \multirow{2}{*}{ Sexo } & Hombres & 149 & $48,8 \%$ \\
\hline & Mujeres & 142 & $51,2 \%$ \\
\hline \multirow{6}{*}{ Nivel de educación } & Primaria incompleta & 39 & $13,4 \%$ \\
\hline & Primaria completa & 38 & $13,1 \%$ \\
\hline & Secundaria incompleta & 65 & $22,3 \%$ \\
\hline & Secundaria completa & 95 & $32,6 \%$ \\
\hline & Superior incompleto & 30 & $10,3 \%$ \\
\hline & Superior completa & 24 & $8,2 \%$ \\
\hline \multirow{5}{*}{ Tiempo de trabajo } & 1 - 2 semanas & 4 & $1,4 \%$ \\
\hline & 3 semanas - 1 mes & 5 & $1,7 \%$ \\
\hline & 2 - 12meses & 32 & $11 \%$ \\
\hline & 1 año - 3 años & 41 & $14,1 \%$ \\
\hline & Más de 3 años & 209 & $71,9 \%$ \\
\hline \multirow{3}{*}{ Edad } & 18 - 35años & 123 & $42,3 \%$ \\
\hline & 36 - 54 años & 111 & $38,1 \%$ \\
\hline & 55 - 78 años & 67 & $19,6 \%$ \\
\hline Total & & 291 & $100 \%$ \\
\hline
\end{tabular}


Tabla 2. Conocimientos de los comerciantes ambulantes de un mercado en Lambayeque, Chiclayo - setiembre, 2017.

\begin{tabular}{|c|c|c|c|c|c|}
\hline & & Correcta & $\%$ & Incorrecta & $\%$ \\
\hline \multirow{4}{*}{$\begin{array}{l}\text { Enfermedad por } \\
\text { exposición solar } \\
\text { prolongada }\end{array}$} & Estrías & 12 & $4,12 \%$ & 279 & $95,88 \%$ \\
\hline & Cáncer de piel & 262 & $90,03 \%$ & 17 & $9,07 \%$ \\
\hline & Sarna & 11 & $3,78 \%$ & 268 & $96,22 \%$ \\
\hline & Celulitis & 6 & $2,06 \%$ & 273 & $97,94 \%$ \\
\hline \multirow{4}{*}{$\begin{array}{l}\text { Característica del } \\
\text { envejecimiento de la } \\
\text { piel }\end{array}$} & Verrugas, lunares & 39 & $13,4 \%$ & 240 & $86,6 \%$ \\
\hline & Acné, ronchas & 33 & $11,3 \%$ & 246 & $88,7 \%$ \\
\hline & Manchas, arrugas & 156 & $53,6 \%$ & 135 & $46,3 \%$ \\
\hline & Picazón, sarpullido & 63 & $21,6 \%$ & 228 & $78,4 \%$ \\
\hline \multirow{4}{*}{$\begin{array}{l}\text { Daños a los ojos por } \\
\text { exposición solar }\end{array}$} & Estrabismo & 7 & $2,4 \%$ & 284 & $97,6 \%$ \\
\hline & Sangrado en los ojos & 21 & $7,2 \%$ & 270 & $92,8 \%$ \\
\hline & Miopía & 100 & $34,36 \%$ & 191 & $65,64 \%$ \\
\hline & Cataratas & 163 & $56,01 \%$ & 128 & $43,99 \%$ \\
\hline \multirow{4}{*}{$\begin{array}{l}\text { Horario donde los rayos } \\
\text { del sol son más fuertes }\end{array}$} & Entre las 10 am y $3 p m$ & 234 & $80,41 \%$ & 57 & $19,59 \%$ \\
\hline & Antes de las 7 am & 6 & $2,06 \%$ & 285 & $97,94 \%$ \\
\hline & Entre las $3 p m$ y $6 p m$ & 27 & $9,27 \%$ & 264 & $90,73 \%$ \\
\hline & Entre las 7am y $10 \mathrm{am}$ & 24 & $8,24 \%$ & 267 & $91,76 \%$ \\
\hline \multirow{4}{*}{$\begin{array}{l}\text { Lo cierto con respecto a } \\
\text { los rayos del sol }\end{array}$} & En un día nublado no te puedes quemar & 48 & $16,49 \%$ & 243 & $83,51 \%$ \\
\hline & $\begin{array}{l}\text { La gente de piel blanca es más sensible a } \\
\text { los rayos del sol }\end{array}$ & 154 & $52,92 \%$ & 137 & $47,08 \%$ \\
\hline & $\begin{array}{l}\text { Si estoy bajo el sol y no siento calor, no me } \\
\text { quemaré }\end{array}$ & 40 & $13,74 \%$ & 251 & $86,26 \%$ \\
\hline & El sol en el invierno no es peligroso & 49 & $16,83 \%$ & 242 & $83,17 \%$ \\
\hline
\end{tabular}

Tabla 3. Hábitos de exposición solar de comerciantes ambulantes que usan bloqueador solar.

\begin{tabular}{|c|c|c|c|}
\hline & & $\mathbf{n}$ & $\%$ \\
\hline \multirow{5}{*}{ ¿Quién le recomendó su uso? } & Médico & 15 & $20,8 \%$ \\
\hline & Enfermera & 3 & $4,2 \%$ \\
\hline & Familiar & 46 & $63,9 \%$ \\
\hline & Farmacéutico/boticario & 5 & $6,9 \%$ \\
\hline & Otro & 3 & $4,2 \%$ \\
\hline \multirow{3}{*}{ ¿Cuántas veces se lo aplica? } & De 1 a 2 veces & 68 & $94,4 \%$ \\
\hline & De 3 a 4 veces & 1 & $1,4 \%$ \\
\hline & De 5 a 6 veces & 3 & $4,2 \%$ \\
\hline \multirow{3}{*}{ ¿Cuándo lo utiliza? } & Solo los días soleados & 24 & $33,3 \%$ \\
\hline & Solo cuando voy a trabajar al mercado & 28 & $38,9 \%$ \\
\hline & Todos los días & 20 & $27,8 \%$ \\
\hline \multirow{3}{*}{ ¿Dónde se lo aplica? } & Solo en cara & 17 & $23,6 \%$ \\
\hline & Cara y cuello & 17 & $23,6 \%$ \\
\hline & Cara, cuello, orejas y manos & 38 & $52,8 \%$ \\
\hline \multirow{3}{*}{ ¿Dónde consigue bloqueador solar? } & Farmacia/botica & 35 & $48,6 \%$ \\
\hline & Supermercado & 1 & $1,4 \%$ \\
\hline & Catálogo & 36 & $50,0 \%$ \\
\hline
\end{tabular}


Tabla 4. Prácticas de fotoprotección, según la escala de Likert en comerciantes ambulantes.

\begin{tabular}{lcccccccccc} 
& \multicolumn{2}{c}{ Siempre } & \multicolumn{2}{c}{ Habitualmente } & \multicolumn{2}{c}{ A veces } & \multicolumn{2}{c}{ Casi nunca } & \multicolumn{2}{c}{ Nunca } \\
& $\mathbf{n}$ & $\%$ & $\mathbf{n}$ & $\%$ & $\mathbf{n}$ & $\%$ & $\mathbf{n}$ & $\%$ & $\mathbf{n}$ & $\%$ \\
\hline Uso sombrilla & 132 & $45 \%$ & 25 & $9 \%$ & 52 & $18 \%$ & 23 & $8 \%$ & 59 & $20 \%$ \\
Usa lentes & 32 & $11 \%$ & 14 & $5 \%$ & 48 & $16 \%$ & 21 & $7 \%$ & 176 & $60 \%$ \\
Usa gorra & 105 & $36 \%$ & 39 & $13 \%$ & 63 & $22 \%$ & 4 & $1 \%$ & 80 & $27 \%$ \\
Usa manga larga & 65 & $22 \%$ & 40 & $14 \%$ & 114 & $39 \%$ & 31 & $11 \%$ & 41 & $14 \%$ \\
Usa pantalón & 177 & $61 \%$ & 42 & $14 \%$ & 56 & $19 \%$ & 4 & $1 \%$ & 12 & $4 \%$ \\
Usa ropa clara & 61 & $21 \%$ & 74 & $25 \%$ & 119 & $41 \%$ & 31 & $11 \%$ & 6 & $2 \%$ \\
\hline
\end{tabular}

Alrededor de un mercado de Chiclayo, setiembre 2017.

\section{DISCUSIÓN}

Muchos agentes afectan la transmisión de la luz ultravioleta a la piel humana. Estos incluyen agentes fotoprotectores naturales (ozono, contaminantes, nubes y niebla), agentes biológicos naturales (cromóforos epidérmicos), agentes fotoprotectores físicos (ropa, sombreros, maquillajes, gafas de sol y vidrios para ventanas) y filtros de luz ultravioleta (ingredientes de protección solar y agentes de bronceado sin sol). Además, existen agentes que pueden modular los efectos de la luz ultravioleta en la piel (antioxidantes y otros). Todo lo anterior se revisa en este artículo ${ }^{(13)}$, aplicado a los vendedores ambulantes o vendedores callejeros.

Encontramos que los comerciantes respondieron algunas preguntas correctas sobre los daños causados por exposición solar. Aproximadamente la mitad de los encuestados respondieron de modo correcto por lo menos cuatro preguntas de un total de cinco, correspondientes a la sección conocimientos, esto esta relación con la exposición al sol que es la principal causa de fotocarcinogénesis, fotoenvejecimiento y fotosensibilidad ${ }^{(14)}$, nuestros resultados muestran que una gran mayoría de los vendedores conoce que el la exposición solar prolongada causa cáncer de piel, esto se corrobora con otros estudios ${ }^{(2,4)}$, además conocen que causa manchas y arrugas llamado fotoenvejecimiento ${ }^{(18)}$.

Para las preguntas sobre enfermedad por exposición prolongada y horarios de mayor radiación solar, la mayoría respondieron correctamente, mientras que, para las otras tres preguntas, poco más de la mitad lo hizo de manera correcta. Rosso et al. menciona que la exposición al sol intermitente e intensa, como durante las vacaciones en la playa, aumenta el riesgo de melanoma maligno (CMM) y carcinoma basocelular $(B C C)$, mientras que la exposición prolongada al sol, como durante las ocupaciones al aire libre, no se asoció con $\mathrm{CMM}$ o $\mathrm{BCC}^{(15)}$.

Al momento del estudio el 71,9 \% llevaban más de 3 años trabajando como comerciantes ambulantes. No se encontraron comerciantes ambulantes entre las edades de 24-36 años. Los resultados más relevantes de las preguntas dirigidas sobre las nociones de los comerciantes en relación con los efectos causados por la radiación solar, en el ítem enfermedad por exposición solar prolongada son que un $90 \%$ reconoce que causa cáncer de piel, este resultado concuerda con los estudios de Montserrat et al. y Castanedo-Cazares et al. donde afirman que la mayor parte de los encuestados reconocióla relación entre radiación ultravioletay cáncer de piel| ${ }^{(6,7)}$, un 53,6\% reconoce que una característica del envejecimiento de la piel por radiación son las manchas y verrugas, en el ítem enfermedad el $90 \%$ reconoce que causa cáncer de piel, resultado similar a lo reportado por Castanedo-Cáceres ${ }^{(7)}$, el ítem de daño a los ojos por exposición solar un 56\% respondió que la radiación solar causa cataratas, en lo que también concluye un estudio en el que se evalúa la carcinogénesis por radiación ultravioleta ${ }^{(9)}$, también el 52,92\% respondió que la gente de piel blanca es más sensible a los rayos del sol, esto se estudió en los fototipos de piel de los ambulantes que trabajan alrededor de un mercado, en la que se observó la siguiente frecuencia: fototipo I $(0,3 \%)$ siempre se quema y nunca se broncea, fototipo II $(4,5 \%)$ se quema con facilidad y se broncea mínimamente, fototipo III $(24,7 \%)$, fototipo IV $(48,8 \%)$, fototipo V $(19,6 \%)$ se queman rara vez y se broncean intensamente, fototipo VI $(2,15)$. En contraste un estudio español de Peña Ortega indica que el fototipo III $(37,23 \%)$ representa la mayoría y le sigue el fototipo IV, que se quema muy poco y se broncea con facilidad $(31,08 \%)^{(10)}$. Solorzano en un estudio en el cono sur de Lima, Perú encontró los fototipo IV en $77,9 \%$, fototipo III en $11,3 \%$, fototipo $\mathrm{V}$ en $9,9 \%$, y fototipo I, II y VI en 
0,9\% que se asemejan a los resultados encontrados en este estudio ${ }^{(16)}$. El 59,5\% de los comerciantes (173/291) señaló acudir a laborar de lunes a domingo, un 39,4\% trabaja de 5-6 días a la semana, y un 4,1\% trabaja 4 o menos días a la semana. Donde aproximadamente un (48,5\%) de ellos declararon exponerse más de 6 horas, un $24,7 \%$ se expone a los rayos solares de $3-6$ horas al día, mientras que el 8,6\% registraron el menor tiempo de exposición solar ( 0 - 2 horas). El concepto de fototipo de piel es práctico y útil para predecir la sensibilidad del individuo a los rayos UV, los factores de riesgo y preventivos, y elegir protectores solares incluso con la limitación ${ }^{(17)}$. La mayoría de los comerciantes no utiliza bloqueador solar (75,3\%), esto representa un grave riesgo para la salud ya que según Castanedo-Cazares el fototipo III presentará quemadura solar con 22-33 min de exposición en un día de verano, mientras que el $\mathrm{V}$ requiere más de $1 \mathrm{~h}$ de exposición ${ }^{(8)}$, se encontró también que solo 72 comerciantes utilizaban protector solar al momento de ser encuestados. El uso regular de protectores solares previene el desarrollo de queratosis solares y, por implicación, posiblemente reduce el riesgo de cáncer de piel a largo plazo(18).

Solo el 24,7\% de los encuestados (72) manifestó utilizar este producto como medida de fotoprotección; de ellos, el 63,9\% (68) manifestaron que un familiar les recomendó su uso, este dato se contrasta con el estudio de Molgó donde el $72 \%$ de la población reconoció haber recibido información sobre prevención de cáncer de piel, siendo su principal fuente de información fue la televisión $(57,7 \%)$, mientras los familiares $(5,7 \%)^{(6)}$. El $94,4 \%$ lo usa de $1-2$ veces al día, el $38,9 \%$ solo se lo aplica cuando va a laborar, 52,8\% (38/72) y lo usa en cara, cuello, orejas y manos, este comportamiento es de riesgo ya que Sordo aplicarse un fotoprotector treinta minutos antes de salir y reaplicarse cada una o dos horas, según la actividad deportiva o laboral a realizar ${ }^{(19)}$, esto debería ser mínimo con protectores SPF 30+(24), y la mitad de ellos (50\%) lo obtiene de un catálogo. Los hábitos más comunes utilizados como práctica de fotoprotección fueron utilizar sombrillas (45\%) y pantalones (61\%), mientras que los menos empleados fueron el uso de lentes (60\%) y gorras (27\%). Las medidas de comportamiento, por ejemplo, usar ropa protectora contra el sol y un sombrero y reducir al mínimo la exposición al sol, deben preferirse a los protectores solares ${ }^{(14)}$. Nuestro trabajo contribuye principalmente en la identificación de quienes han de ser los principales destinatarios de las campañas, sobre todo gente que tiene un bajo conocimiento sobre radiación solar en la salud, dado que son los que más se exponen y padecen los efectos de la toxicidad solar aguda. Sin embargo, incluso con suficientes métodos de prevención, la falta de educación y la promoción de una práctica no conducirá a resultados favorables ${ }^{(1)}$.

Las limitaciones del estudio se vieron afectadas por los días de muestreo ya que el trabajo de los ambulantes es muy variable, asistiendo a las inmediaciones del mercado no de manera diaria, si no interdiario o teniendo sus propios horarios lo que no permitió encuestar a la mayoría de ambulantes. El tamaño de muestra fue reducido pudiendo agregar a los ambulantes de otros mercados como por ejemplo de los centros comerciales y distintas calles de la ciudad. Tuvimos una limitación en el estudio ya que no validamos anteriormente nuestro cuestionario. Concluimos con que los conocimientos y hábitos de exposición solar en los comerciantes ambulantes son de regular a adecuado y los hábitos deficientes.

\section{CONCLUSIÓN}

Nuestro trabajo contribuye principalmente en la identificación de quienes han de ser los principales destinatarios de las campañas, sobre todo gente que tiene un bajo conocimiento sobre radiación solar en la salud, dado que son los que más se exponen y padecen los efectos de la toxicidad solar aguda. Sin embargo, incluso con suficientes métodos de prevención, la falta de educación y la promoción de una práctica no conducirá a resultados favorables ${ }^{(1)}$.

Contribuciones de autoría: Los autores participaron en la génesis de la idea, diseño de proyecto, recolección e interpretación de datos, análisis de resultados y preparación del manuscrito del presente trabajo de investigación.

Financiamiento: Autofinanciado.

Conflicto de interés: Los autores declaran no tener conflicto de interés en la publicación de este artículo.

Recibido: 09 de marzo 2020

Aprobado: 30 de marzo 2020

Correspondencia: Sebastian Iglesias-Osores.

Dirección: Huamachuco, Lambayeque 14013 - Perú.

Teléfono: (074) 283146

Correo:sebasiglo@gmail.com, siglesias@unprg.edu.pe 


\section{REFERENCIAS BIBLIOGRÁFICAS}

1. Queen L. Skin Cancer: Causes, Prevention, and Treatment. Sr Honor Theses. 2017 Apr. Disponible en: https://digitalcommons.liberty.edu/honors/648/

2. Lake E. Trends of skin cancer mortality after transplantation in the United States: 1987 to 2013. J Am Acad Dermatol. 2018 Dec. DOI: 10.1016/j. jaad.2016.02.1155

3. Piñeros M, Ramos W, Antoni S, Abriata G, Medina LE, Miranda JJ, et al. Cancer patterns, trends, and transitions in Peru: a regional perspective. Lancet Oncol. 2017 Oct;18(10):e573-86. DOI: 10.1016/S1470-2045(17)30377-7

4. Sandby-Møller J, Poulsen T, Wulf HC. Epidermal Thickness at Different Body Sites: Relationship to Age, Gender, Pigmentation, Blood Content, Skin Type and Smoking Habits. Acta Derm Venereol [Internet]. 2003 [cited 2020 Mar 20];83(6):410-3. DOI: 10.1080/00015550310015419

5. Borschmann RD, Cottrell D. Developing the readiness to alter sun-protective behaviour questionnaire (RASP-B). Cancer Epidemiol [Internet]. 2009 Dec [cited 2020 Mar 20];33(6):451-62. DOI: 10.1016/j.canep.2009.09.003

6. Montserrat Molgó N, Celso Castillo A, Valdés F R, Romero G W, Jeannere $M$ V Cevo $E$ T et al. Conocimientos y hábitos de exposición solar de población chilena. Rev Med Chil. 2005 Jun;133(6):662-6. DOI: 10.4067/S003498872005000600007.

7. Castanedo-Cazares JP, Torres-Álvarez B, Medellín-Pérez ME, AguilarHernández GA, Moncada B. Conocimientos y actitudes de la población mexicana con respecto a la radiación solar. Gac Med Mex. 2006;142(6):4515. Disponible en: https://www.medigraphic.com/cgi-bin/new/resumen cgi?IDARTICULO=13466

8. Cázares JPC, Álvarez BT, Ondarza SS, Pérez AE, Moscoso AG. Estimación de tiempo de exposición solar para quemadura en población mexicana. Gac Med Mex. 2012;148(3):343-7. Disponible en: https://www.anmm.org.mx/ GMM/2012/n3/GMM_148_2012_3_243-247.pdf

9. Nishigori C, Yarosh DB, Donawho C, Kripke ML. The immune system in ultraviolet carcinogenesis. J Investig dermatology Symp Proc 1996 Apr;1(2):143-6. Disponible en: https://www.ncbi.nlm.nih.gov/ pubmed/9627708
10. Peña Ortega M, Buendía Eisman A, Ortega del Olmo R, Serrano Ortega S. Hábitos de fotoprotección en la Facultad de Ciencias de la Educación Física y el Deporte de la Universidad de Granada. Piel. 2013 Jan;19(4):179-83. DOI: 10.1016/S0213-9251(04)72828-2

11. Fajre $X$, Barría $K$, Muñoz C. Exposición solar y Fotoprotección. Rev Chil Med Fam. 2017 Aug:3(3):113-8. Disponible en: http://www. revistachilenademedicinafamiliar.cl/index.php/sochimef/article/view/308

12. Aguilar Casana LE, Marcelo Sandoval JE. Nivel de conocimiento sobre cáncer de piel y la práctica de medidas preventivas en vendedores ambulantes. Centro histórico de Trujillo, 2014. Universidad Privada Antenor Orrego. Universidad Privada Antenor Orrego - UPAO; 2015. Disponible en: http://repositorio.upao.edu.pe/handle/upaorep/1668

13. Kullavanijaya P, Lim HW. Photoprotection. J Am Acad Dermatol. 2005 Jun 1;52(6):937-58. DOI: 10.1016/j.jaad.2004.07.063

14. Lautenschlager S, Wulf HC, Pittelkow MR. Photoprotection. Vol. 370, Lancet. Elsevier; 2007. p. 528-37. DOI: 10.1016/S0140-6736(07)60638-2.

15. Rosso S, Zanetti R, Pippione M, Sancho-Garnier H. Parallel risk assessment of melanoma and basal cell carcinoma: Skin characteristics and sun exposure Melanoma Res [Internet]. 1998 Dec 1 [cited 2020 Mar 20];8(6):573-83. DOI: 10.1097/00008390-199812000-00013

16. Solórzano S, Contreras G, Pérez C. Aspectos epidemiológicos y percepciones del acné vulgar en escolares de secundaria del Cono Sur de Lima - Perú. Folia dermatol. 2005;16(3):113-8. Disponible en: http://bases.bireme.br/

Kawada A. Risk and preventive factors for skin phototype. J Dermatol Sci. 2000 Mar 1;23(SUPPL. 1):S27-9. DOI: 10.1016/S0923-1811(99)00074-2

18. Thompson SC, Jolley D, Marks R. Reduction of Solar Keratoses by Regular Sunscreen Use. N Engl J Med [Internet]. 1993 Oct 14 [cited 2020 Mar 20];329(16):1147-51. DOI: 10.1056/NEJM199310143291602

19. Sordo C, Gutiérrez C. Skin cancer and sun radiation: Peruvian experience in the prevention and early detection of skin cancer and melanoma. Rev Peru Med Exp Salud Publica. 2013 Mar;30(1):113-7. DOI: 10.1590/s172646342013000100021 